\title{
An 84-year-old man with lower extremity bullae
}

\author{
Katherine Dittman, Christopher Jake Williams, Craig Rohan \\ Wright Patterson Medical Center, Ohio, United States
}

Received: May 1, 2018

Accepted: May 27, 2018

Online Published: June 7, 2018

DOI: $10.5430 /$ crim.v5n3p4

URL: https://doi.org/10.5430/crim.v5n3p4

\begin{abstract}
Deep vein thromboses (DVT) are often associated with post-thrombotic syndrome (PTS) and long term side effects such as chronic venous insufficiency or venous stasis ulcers, but these symptoms typically develop months-to-years after presentation of the DVT. Here, we report a case of severe lower extremity bullae and ulcers one week after diagnosis and treatment of DVT.
\end{abstract}

Key Words: Venous thrombosis, Thromboembolism, Blister, Ulcer, Hydrostatic pressure

\section{CASE REPORT}

An 84-year-old gentleman with past medical history significant for hypertension, coronary artery disease, and diabetes mellitus type 2 presented with chief complaint of sudden onset left lower extremity swelling and pain with ambulation. Ultrasound revealed a Deep vein thromboses (DVT) extending from common femoral vein to popliteal vein below the trifurcation. He was started on heparin drip secondary to impaired renal function with a creatinine of 1.6 (baseline 1.0). Once his renal function improved with administration of IV fluids, he was switched to apixaban 10mg twice daily for 7 days, then $5 \mathrm{mg}$ twice daily and discharged home. Five days after discharge, he developed distal left leg pain and blisters with skin sloughing. The patient returned to the ED, where one dose of vancomycin was given for possible cellulitis after blood and wound cultures were done. On admission evaluation, the leg did not appear to be acutely infected, but due to concern for superinfection, patient was started on oral clindamycin and topical ketoconazole. Blood cultures and wound cultures were negative. The left lower leg had multiple coalescent bullae, some decompressed (see Figure 1). There was no circumferential erythema. There were no blisters above the popliteal fossa, or on the contralateral leg. Patient had intact pulses, no cyanosis or bluish discoloration of skin. There was no extension of the prior clot, and no evidence of phlegmasia cerulean dolens. The patient was evaluated by wound care, his pain was controlled and he was discharged to a skilled nursing facility for rehabilitation.

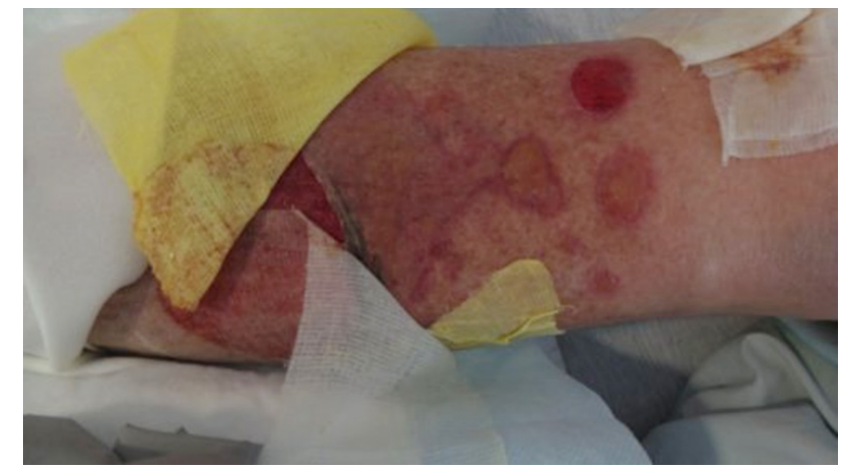

Figure 1. Bullae on left lower extremity

\section{Discussion}

Venous thrombosis is a common occurrence, with an estimated incidence of 1 per 1,000 annually in adult popula-

\footnotetext{
*Correspondence: Katherine Dittman; Email: katie.dittman@gmail.com; Address: Wright Patterson Medical Center 4881 Sugar Maple Dr, Wright-Patterson AFB, Ohio, United States.
} 
tions. ${ }^{[1]}$ Two-thirds of these thrombotic events are DVT. The rate of occurrence continues to climb as a person ages, up to 13-fold greater than those aged 45-55. Unprovoked or idiopathic DVT comprise $25 \%-40 \%$ of all DVT. ${ }^{[1,2]}$

After an initial DVT, post-thrombotic syndrome (PTS) occurs in $20 \%-50 \%$ of patients. ${ }^{[3]}$ PTS is more common in patients with DVT proximal to the knee, are overweight and in those who do not take their anticoagulants or are not within therapeutic levels on anticoagulation. PTS is manifested by venous ulcers, venous insufficiency and discoloration. These symptoms typically develop months-to-years after diagnosis and treatment of DVT. It is posited that chronic venous hypertension leads to symptoms of PTS. ${ }^{[4]}$ PTS is normally treated similarly to other venous insufficiency diseases via compression stockings and symptom management, rarely requiring surgery.

Another complication of DVTS is phlegmasia cerulean dolens, a syndrome associated with cyanosis and increased clot size, leg swelling and pain. This often results in gangrene of the distal limb and further complications, and can be associated with pulmonary embolus.

In this case, a much rarer and more acute symptomatology evolved secondary to acute hydrostatic shifts in the lower

\section{REFERENCES}

[1] White RH. The Epidemiology of Venous Thromboembolism. Circulation. 2003; 107: I-4-I-8. https://doi.org/10.1161/01.CIR .0000078468 .11849 .66

[2] Cushman M. Epidemiology and Risk Factors for Venous Thrombosis. Seminars in Hematology. 2007; 44(2): 62-69. PMid:17433897. https://doi.org/10.1053/j.seminhematol.2007.02.004

[3] Vazquez SR, Kahn SR. Postthrombotic Syndrome. Circulation. 2010; 121: e217-e219. https://doi.org/10.1161/CIRCULATIONAHA .109 .925651 extremity resulting in the formation of large sterile bullae. Edema and swelling of the thigh, coupled with the blockage of a large vein, caused blistering and bullae through rapidly increased hydrostatic pressures. Normally, blisters form over time with friction or increased pressure; ${ }^{[5]}$ however, in this case, the acute change in venous return caused an acute change in interstitial fluid hydrostatic pressure. This resulted in separation of epidermal cells at the level of the stratus spinosum, leading to extensive large bullae, ${ }^{[6]}$ many of which burst with weeping of serous fluid. This was an interesting and rare example of acute hydrostatic pressures changes with acute venous thrombosis.

\section{COnSEnt}

Consent for publication was provided by the patient.

\section{AUTHOR CONTRIBUTION}

$\mathrm{KD}$ contributed in writing the manuscript and in interpretation of literature. $\mathrm{CW}$ was a major contributor in writing and editing the manuscript. RH contributed imaging and examination of patient. All authors read and approved the final manuscript.

\section{CONFLicts OF INTEREST Disclosure}

The authors declare that they have no competing interests.
[4] Kahn SR, Comerota AJ, Cushman M, et al. The Postthrombotic Syndrome: Evidence-Based Prevention, Diagnosis, and Treatment Strategies. Circulation. 2014; 130: 1636-1661. PMid:25246013. https://doi.org/10.1161/CIR.0000000000000130

[5] Stoughton RB. Mechanisms of Blister Formation. AMA Arch Derm. 1957; 76(5): 584-590. PMid:13468790. https://doi.org/10.1 001/archderm.1957.01550230050008

[6] Knapik JJ, Reynolds KL, Jones BH. Friction blisters. Pathophysiology, prevention and treatment. Sports Med. 1995; 20(3): 136147. PMid:8570998. https://doi.org/10.2165/00007256-1 99520030-00002 